\title{
Analysis of side effect manifestation and coping behavior of rheumatoid arthritis outpatients on intravenous drip and subcutaneous injection treatment by biologics
}

\author{
Akiko Matsuda ${ }^{1}$, Yuki Ikezoe ${ }^{2}$, Junichi Hasegawa ${ }^{3}$, Yusuke Okamoto ${ }^{4}$, Shunsuke Kanayama ${ }^{5}$, \\ Hiroshi Hagino ${ }^{6}$
}

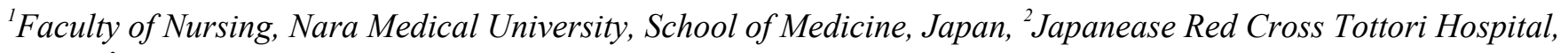
Japan, ${ }^{3}$ Tottori University Faculty of Medicine, Department of Pathophysiological and Therapeutic Science, Japan, ${ }^{4}$ Tottori University Hospital, Japan, ${ }^{5}$ Department of Nursing, The University of Shimane, Japan, ${ }^{6}$ Tottori University Faculty of Medicine School of Health Science, Japan

Background:While rheumatoid arthritis(RA) has become more treatable due to the introduction of biologics(Bio), when side effects occur,they are likely to be severe. Therefore, coping behaviors including pharmacotherapy and infection prevention are required for outpatients in recuperation.

Purpose:We investigated the manifestation of side effects and coping behavior including infection prevention in outpatients on intravenous drip and subcutaneous injection biologics treatments.

Method:The subjects were 58 female RA outpatients over the age of 20 undergoing biologics treatment at T hospital. 23 were part of the intravenous drip group(DIV), and 35 were part of the subcutaneous injection group (SC). We conducted interviews with questionnaires and collected information from medical records. We investigated possible side effects as listed in the package inserts of Bio. Infusion reaction symptoms are defined as side effects during treatment by biologics. Side effect symptoms are defined as undesirable symptoms beyond the infusion reaction. This research was conducted with the approval of the Ethics Committee of T University Faculty of Medicine.

Result: There were no significant group difference in mean age(DIV:65.9,SC:62.8) and measures of daily life activities (DIV:0.85, SC:1.03). In both groups, approximately $10 \%$ of subjects manifested infusion reactions, including dyspnea, dizziness and heat sensation. Symptoms of dry cough and stomatitis manifest significantly more in the DIV. There was no significant difference between both groups, but malaise, dyspnea and edema side effects manifested in approximately half of the patients. More than $80 \%$ of subjects in both groups practiced gargling and washing hands; however, the majority of subjects in both groups did not practice wearing masks and drying hands with alcohol.The majority of subjects in both groups were able to contact the hospital in case of emergency.

Conclusion:There were significantly more people on the drip intravenous treatments who manifested side effects compared to those who underwent subcutaneous injection biologics treatments. The percentage of infusion symptoms regardless of the kind of injection, is $10 \%$. Most of the outpatients didn't apply the appropriate infection prevention behavior. 